\title{
The STIX Aspect System (SAS): The Optical Aspect System of the Spectrometer/Telescope for Imaging X-Rays (STIX) on Solar Orbiter
}

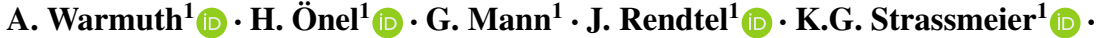 \\ C. Denker ${ }^{1}(D)$ G.J. Hurford ${ }^{2,3}$ • S. Krucker ${ }^{2,3}$ • J. Anderson ${ }^{1,4,5}$ - S.-M. Bauer ${ }^{1}$. \\ W. Bittner ${ }^{1}$ - F. Dionies ${ }^{1}$. J. Paschke ${ }^{1}$ - D. Plüschke ${ }^{1}$ - D.P. Sablowski ${ }^{1}$. F. Schuller ${ }^{1}$ (D)

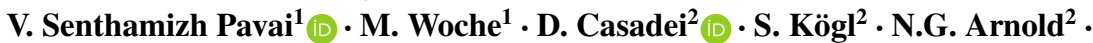 \\ H.-P. Gröbelbauer ${ }^{2}$ - D. Schori ${ }^{2}$ - H.J. Wiehl ${ }^{2}$ - A. Csillaghy ${ }^{2}$. O. Grimm ${ }^{2,6}$. \\ P. Orleanski ${ }^{7}$ (D) K.R. Skup ${ }^{7}$ - W. Bujwan ${ }^{7}$ (D) K. Rutkowski ${ }^{7}$ K. Ber ${ }^{7}$
}

Received: 13 March 2020 / Accepted: 19 June 2020 / Published online: 3 July 2020

(C) The Author(s) 2020

\begin{abstract}
The Spectrometer/Telescope for Imaging X-rays (STIX) is a remote sensing instrument on Solar Orbiter that observes the hard X-ray bremsstrahlung emission of solar flares. This paper describes the STIX Aspect System (SAS), a subunit that measures the pointing of STIX relative to the Sun with a precision of $\pm 4^{\prime \prime}$, which is required to accurately localize the reconstructed X-ray images on the Sun. The operating principle of the SAS is based on an optical lens that images the Sun onto a plate that is perforated by small apertures arranged in a cross-shaped configuration of four radial arms. The light passing through the apertures of each arm is detected by a photodiode. Variations of spacecraft pointing and of distance from the Sun cause the solar image to move over different apertures, leading to a modulation of the measured lightcurves. These signals are used by ground analysis to calculate the locations of the solar limb, and hence the pointing of the telescope.
\end{abstract}

Keywords Instrumentation and data management · Flares, spectrum $\cdot$ X-ray bursts, spectrum · Corona

\footnotetext{
$\triangle$ A. Warmuth

awarmuth@aip.de

1 Leibniz-Institut für Astrophysik Potsdam (AIP), An der Sternwarte 16, 14482 Potsdam, Germany

2 University of Applied Sciences and Arts Northwestern Switzerland, Bahnhofstrasse 6, 5210 Windisch, Switzerland

3 Space Sciences Laboratory, University of California, 7 Gauss Way, 94720 Berkeley, USA

4 Present address: Institut für Geodäsie und Geoinformationstechnik, Technische Universität Berlin, Straße des 17. Juni 135, 10623 Berlin, Germany

5 Present address: GFZ German Research Centre for Geosciences, Telegrafenberg, 14473 Potsdam, Germany

6 Institute for Particle Physics and Astrophysics, ETH Zürich, Otto-Stern-Weg 5, 8093 Zürich, Switzerland

7 Space Research Centre, Polish Academy of Sciences, Bartycka 18A, 00-716 Warszawa, Poland
} 


\section{Introduction}

The ESA-led Solar Orbiter mission addresses the interaction between the Sun and the heliosphere (Müller et al., 2013). Launched in February 2020, the spacecraft will perform multiple encounters with the Sun with closest approaches down to 0.28 astronomical units. Solar Orbiter carries ten instruments which provide both remote sensing and in-situ observations. One of these instruments is the Spectrometer/Telescope for Imaging X-rays (STIX; Krucker et al., 2020) which observes the bremsstrahlung X-ray emission of thermal plasmas and accelerated electrons in solar flares.

STIX is a Fourier-type imager based on a pair of tungsten grid assemblies containing 30 subcollimators which are spatially modulating the incoming X-ray flux. The grid pairs create Moiré patterns which contain encoded information on the location, size, and shape of the $\mathrm{X}$-ray sources. Behind the grid pairs, 32 coarsely pixelated CdTe detectors register energy and timing of the incident X-ray photons. In this way, 30 visibilities are measured that are then used to reconstruct X-ray images with a resolution of $7^{\prime \prime}$ at energies from 4 to $150 \mathrm{keV}$. The final data products include X-ray lightcurves, spectra, and images. Two detectors are dedicated to background monitoring and coarse flare location.

Hard X-ray (HXR) imagers like STIX or its predecessors RHESSI (Lin et al., 2002) and HXT (Kosugi et al., 1991) face challenges concerning alignment and pointing. Image reconstruction relies on the knowledge of the orientation of the collimator pairs relative to the direction to the Sun. Co-alignment with images in other wavelengths is also an issue, since in HXRs only the thermal and nonthermal flare sources are visible, and thus no other solar features that are conventionally used for co-alignment (e.g. the solar limb) can be used. Therefore, HXR imagers usually incorporate a dedicated optical aspect system.

In the case of STIX, the pointing information provided by the spacecraft attitude control system is sufficient for reconstructing images with the specified 7 " resolution. Precise placement of these images on the Sun additionally requires an accurate knowledge of the offset between the STIX imaging axis and the spacecraft reference frame. Due to thermal flexing of the spacecraft panel on which STIX and the other remote sensing instruments are mounted, this relative pointing can only be guaranteed at the $2^{\prime}$ level. A dedicated system is therefore required to provide the pointing information with the required accuracy. The relative stability of the thermal environment suggests that one calibration every few days is sufficient.

The STIX Aspect System (SAS) was designed to reduce the pointing uncertainty with respect to the Sun from the $\pm 2^{\prime}$ that are provided by the spacecraft to $\pm 4^{\prime \prime}$, which enables STIX images to be placed in the context of images acquired in other wavelengths. This is particularly relevant for coordinated studies using the other instruments on Solar Orbiter that are capable of obtaining spatially resolved observations of solar flare signatures, namely PHI (Polarimetric and Helioseismic Imager; Solanki et al., 2020), EUI (Extreme Ultraviolet Imager; Rochus et al., 2020), and SPICE (Spectral Imaging of the Coronal Environment; SPICE Consortium: Anderson et al., 2020). Moreover, locating the X-ray sources is crucial for establishing the magnetic connectivity of the flare site to Solar Orbiter, which is necessary to evaluate the role of the flare as a source of energetic particles that are observed in-situ with EPD (Energetic Particle Detector; Rodriguez-Pacheco et al., 2020).

Note that the SAS plays no role in the image reconstruction itself, and does not measure the roll orientation about Sun center, which are provided by the s/c aspect solution. The expected uncertainties in roll angle translate to a source shift at the limb (where the effect will be strongest) of below $2^{\prime \prime}$ in the worst case, which justifies this reliance on s/c aspect. 


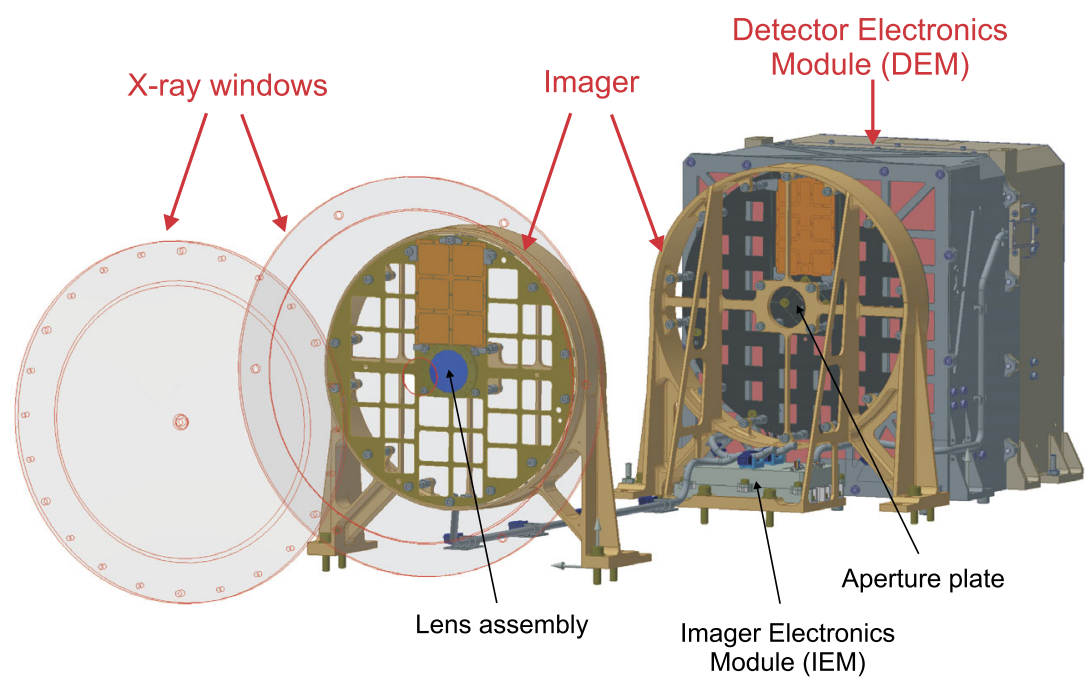

Figure 1 Overview of the STIX instrument hardware. Indicated are the three main mechanical components (X-ray windows, Imager, Detector Electronics Module), as well as major components of the aspect system (lens assembly, aperture plate, Imager Electronics Module).

In Section 2, we describe the design of the SAS and its main components. The data handling and system performance is discussed in Section 3, and the summary is given in Section 4.

\section{Instrument Design and Components}

\subsection{General Design and Working Principle}

The design challenges for the aspect system were manifold. With available resources that only amount to a tiny fraction of a total allocated mass of $7 \mathrm{~kg}$, a power of $8 \mathrm{~W}$, and a telemetry of 700 bits s $^{-1}$ for the whole STIX instrument, the SAS is severely limited in terms of mass, volume, power, data processing requirements and telemetry. Due to the large thermal load near perihelion, the system has to view the Sun through a small aperture. As designed, the operational temperature of the SAS ranges from $-40^{\circ} \mathrm{C}$ up to $85^{\circ} \mathrm{C}$ at perihelion (the non-operational temperature range is from $-55^{\circ} \mathrm{C}$ up to $90^{\circ} \mathrm{C}$ ). This temperature range is significantly broader than typically encountered in missions in Earth orbit, e.g. it is six times broader than was required for the solar aspect system on RHESSI (cf. Zehnder et al., 2003). Crucially, the system has to account for a solar angular diameter that is varying by factor of 4 during the mission due to the highly elliptical orbit (closest perihelion: $0.284 \mathrm{AU}$, farthest aphelion: $1.02 \mathrm{AU}$ ). Naturally, the system has to withstand the environmental conditions both during launch (vibrations, acoustic) and in flight (radiation, contamination, thermal effects, ageing).

These requirements imply the need for a simple and robust aspect system with a large performance margin. A conventional aspect system based on a telescope imaging the Sun onto a CCD chip or linear diode arrays was found not to be feasible given the constraints. Thus we have developed an innovative design for an aspect system that relies on extracting the aspect solution from time series of photometric measurements. 


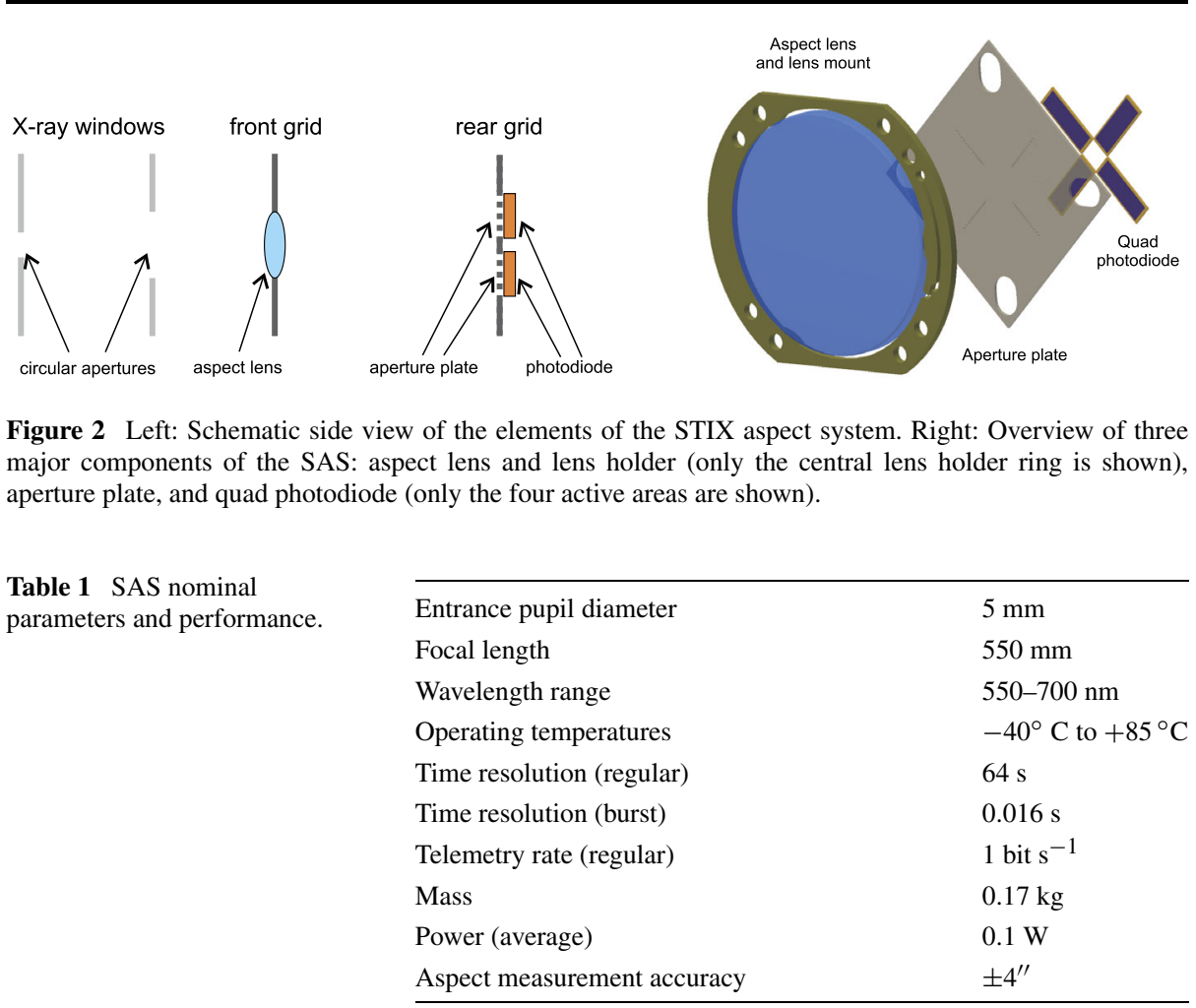

Mechanically, the STIX instrument consists of three main parts (see Figure 1):

i) X-ray entrance windows: a spacecraft-provided feedthrough integrated in Solar Orbiter's heat shield containing two beryllium windows. It provides thermal protection against the intense radiative flux near perihelion.

ii) Imager: a pair of tungsten grid assemblies with individual mounts required for Fourier imaging. The imager also contains the main components of the SAS.

iii) Detector/Electronics Module (DEM): contains the X-ray detectors, front-end and digital electronics, power supply and interfaces to the spacecraft.

Major SAS components are indicated in Figure 1, and are also shown in the sketch presented in the left panel of Figure 2 for clarity. The SAS views the Sun through two small holes in the X-ray windows of STIX. An optical lens mounted in the center of the front grid assembly focuses an image of the Sun onto the rear grid. There, the grid is perforated by small apertures which are arranged in a cross-shaped configuration of four radial arms. The light passing through the apertures of each arm induces a current in a photodiode mounted behind this aperture plate. Changes of the spacecraft pointing and the varying distance from the Sun cause the solar image to move over different apertures, resulting in a modulation of the measured lightcurves. These signals are used by ground analysis to calculate the locations of the solar limb, and hence the pointing of the telescope. The basic parameters of the SAS are summarized in Table 1.

We stress that by integrating the critical aspect system elements (lens and apertures) into the grids themselves, the positions of the lens center and apertures relative to the X-ray- 

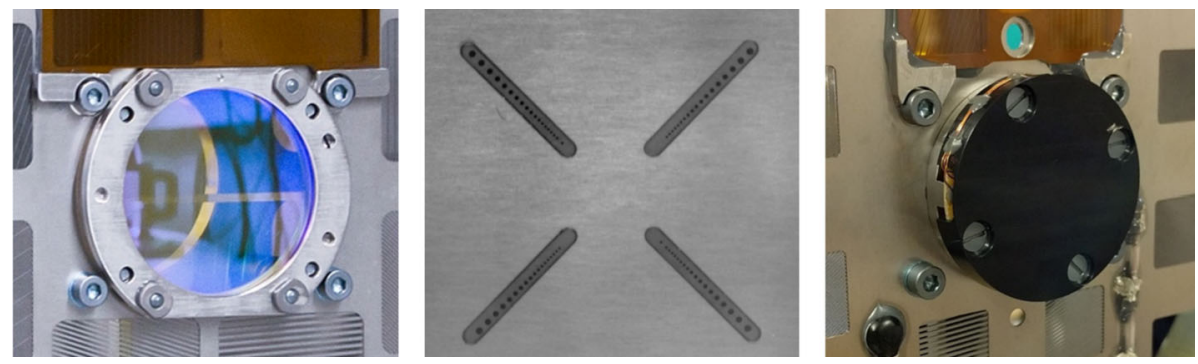

Figure 3 Photos of the main SAS hardware components on the flight unit of STIX. Left: the aspect lens integrated in the front grid. Note the bar behind the lens, which forms an integral part of the front grid and allows one to measure the relative twist between front and rear grid. Middle: the aperture plate integrated in the rear grid as seen through the aspect lens. Right: the Imager Diode Mount (IDM) attached to the backside of the rear grid. The cable at the lower right connects to the Imager Electronics module (IEM) and transmits the photodiode signals.

transmitting grids are accurately known. The aspect calibration is therefore independent of the mechanical properties of the grid mounts and the s/c mounting plate.

\subsection{SAS Components}

In the following, we describe the design of the main hardware components of the SAS.

\subsubsection{Apertures in the X-Ray Entrance Window}

The two beryllium windows both contain a central hole (with a diameter of $5 \mathrm{~mm}$ and $25 \mathrm{~mm}$ for the outer and inner window, respectively). The SAS views the Sun through these holes. The diameter of the outer hole was chosen in order to minimize heat input (a total power of $0.33 \mathrm{~W}$ enters the instrument at perihelion) while at the same time providing adequate diffraction performance. The inside of the $5 \mathrm{~mm}$ aperture in the front window is tapered by 2 degrees to eliminate undesirable solar reflections into the aspect lens.

\subsubsection{Aspect Lens and Lens Mount}

The center of the front grid assembly contains the aspect lens. This is a singlet plano-convex fused silica lens (manufactured from radiation-hard SQ1 glass) with an effective diameter of $29 \mathrm{~mm}$ and a focal length of $550 \mathrm{~mm}$. The resulting plate scale on the rear grid is $0.375^{\prime \prime} \mu \mathrm{m}^{-1}$, leading to a solar image diameter which varies between $18 \mathrm{~mm}$ (for a perihelion of $0.284 \mathrm{AU}$ ) and $5.1 \mathrm{~mm}$ (for a distance of $1 \mathrm{AU}$ ). For reference, the requirement of an aspect accuracy of $4^{\prime \prime}$ translates to a position accuracy in the image plane of $10.7 \mu \mathrm{m}$.

Both sides of the lens are coated with reflective $\mathrm{TiO} 2 / \mathrm{SiO} 2$ layers which act as a bandpass filter that transmits only the wavelength range of 550-700 $\mathrm{nm}$. This reduces the heat load for the Imager and also limits chromatic aberration. The coated lens was designed and manufactured by the Fraunhofer Institute for Applied Optics and Precision Engineering IOF Jena (Germany). Similar lenses and coatings have been flown on previous space missions.

The lens is attached to the front grid via a lens mount consisting of three titanium rings. Two outer rings fixate the lens with respect to the direction normal to the grid, while an inner ring fixates the lens in the grid plane (cf. right panel of Figure 2). This is accomplished via two hard stops and one flexible stop attached to a spring. This ensures a secure mounting 
Figure 4 Geometry of the aperture plate as seen from the detector (i.e., looking sunwards). The mounting plate is in the -Y direction. For reference, the solar limb in the image plane is plotted for the minimum perihelion distance of $0.284 \mathrm{AU}$ and for $1 \mathrm{AU}$.

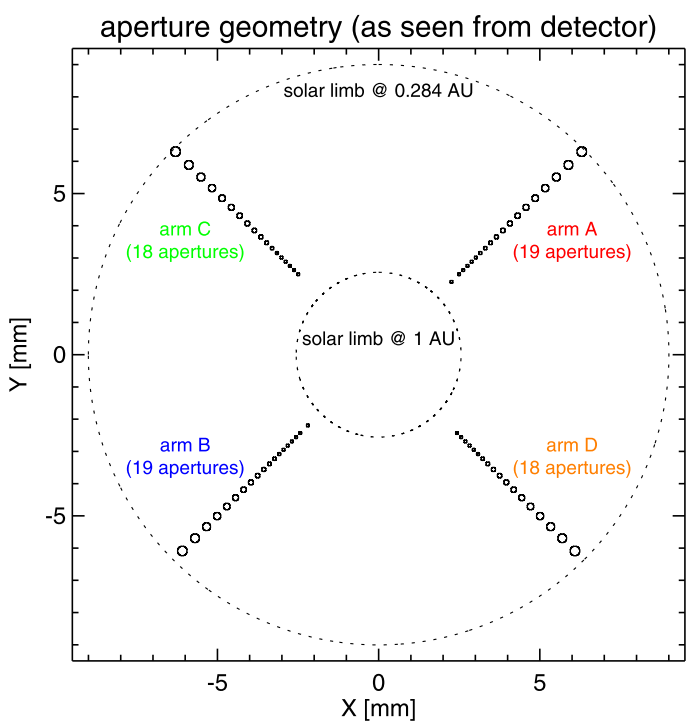

and compensates for variations of the lens diameter due to thermal expansion. This is crucial due to the wide temperature range of $95 \mathrm{~K}$ that will be encountered in flight. The same effect has also to be taken into account when fixing the lens assembly to the front grid. This has been accomplished by kinematic mounts, which account for differences of thermal expansion between the tungsten grid and the lens mount. The lens mount also includes three fiducials which are used to characterize the position of the lens mount with respect to the grid assembly. Prior to integration, the location of the optical center of the lens was measured to a precision of $\pm 5 \mu \mathrm{m}$. Figure 3 (left) shows the fully integrated aspect lens assembly on the front grid of the STIX flight model.

\subsubsection{Aperture Plate}

The aperture plate is located in the center of the rear grid (see Figures 2 and 3). It is a $0.1 \mathrm{~mm}$ thick tungsten plate with a cross-shaped arrangement of tiny apertures. The four orthogonal radial arms of the cross are labeled A, B, C, and D (see Figure 4). The aperture plate was manufactured by FHNW (Switzerland) by laser-cutting and has been integrated into the grid assembly during the actual grid manufacturing process. The sizes and positions of the apertures were precisely measured (at the $2 \mu \mathrm{m}$ level) during the characterization of the rear grid assembly by $2 \mathrm{D}$ optical scanning.

Each of the four arms consists of a series of circular holes. Two of the arms (C and D) have 18 holes, while the other two arms (A and $\mathrm{B}$ ) have an additional aperture closer to the center of the cross. The reason for the unequal number of apertures is historical and was mandated by the original photodiode layout. The diameters of the apertures are increasing from $90 \mu \mathrm{m}$ (innermost apertures) to $300 \mu \mathrm{m}$ (outermost apertures). This guarantees that the signal increases resulting from the solar image covering successive apertures is always be larger than $20 \%$ of the total signal. This was adopted as a safeguard against signal-to-noise issues resulting from the varying heliocentric distance. Going radially outwards, the distance between the consecutive apertures in each arm increases in the same manner. This ensures that the time step between aperture crossings of the solar limb stays roughly constant during the elliptical orbit. 
The radial distances of the outermost apertures from the center of the aperture cross was chosen so that all apertures are covered by the solar image at the closest perihelion. The radial distances of the innermost apertures were constrained by the photodiode geometry.

\subsubsection{Photodiode and Imager Diode Mount}

The light passing through each of the four arms of the aperture plate is directly recorded by a quad photodiode (manufactured by Opto Diode Corp., USA) which is hermetically sealed with a glass cap. The photodiode is electronically similar to Opto Diode's family of UV Enhanced Detectors (UVG) photodiodes which feature stable responsivity over a wide temperature range. Similar photodiodes have been flown on NASA's EOS and SORCE missions.

Each of the four active areas of the diode is located behind an aperture arm (cf. Figure 2). The quad diode is soldered to a printed circuit board (PCB) and attached to the rear grid by a cylindrical housing and a ring, which together form the Imager Diode Mount (IDM; see Figure 3).

\subsubsection{Electronics: Imager Electronics Module and Instrument Data Processing Unit}

The four signals produced by the quad photodiode are fed to a PCB board located in the Imager Electronics module (IEM) which is attached to the rear mount of the Imager (see Figure 1). The board was designed by CBK Warsaw (Poland) and assembled by Matra (France). In the IEM, the analog photodiode signals are first amplified and then digitized by two 12-bit A/D converters (ADCs). For redundancy, one ADC processes the signals from the orthogonal arms A and D, the other for arms B and C. The photodiode is read out repetitively, thus generating four time series of data. The IEM also receives and processes the signals of eight thermal sensors. The digital signals are transferred to the Instrument Data Processing Unit (IDPU; see Skup et al., 2012), a subunit of the DEM (cf. Figure 1), for further processing and storage.

\subsubsection{Twist Monitoring System}

The SAS has a secondary function while STIX is on ground: it is used as a Twist Monitoring System (TMS) which allows one to measure the relative twist between front and rear grid assembly. The requirement for the relative twist is less than $\pm 1 \mathrm{mrad}$ since larger twists lead to a decrease of the $\mathrm{S} / \mathrm{N}$ ratio in the X-ray images. To perform the measurement, an external camera is placed in front of the Imager and obtains images of the aperture plate in the rear grid through the aspect lens. Then an additional lens introduced into the optical path of the TMS shifts the focus from the rear grid to a bar behind the aspect lens on the front grid (see Figure 3). This bar is an integral part of the front grid assembly. During optical characterization of the grids, the position of the bar and the apertures have been precisely measured with respect to the subcollimators. Measuring the orientation of the bar and the aperture cross thus yields the relative twist between front and rear grid.

The twist measurement was carried out repeatedly during the integration and the environmental testing of STIX. The final measurement prior to instrument delivery has verified that the requirement of less than $\pm 1 \mathrm{mrad}$ relative twist is well fulfilled. 


\section{Aspect Data Handling and System Performance}

\subsection{Expected Signal Levels}

With the knowledge of the distance from the Sun, the solar spectral irradiance, the diameter of the hole in the front X-ray window, the focal length and transmission profile of the aspect lens, the diameters of the apertures, and finally the responsivity of the diode, the expected signal levels can be computed. For a perihelion of $0.284 \mathrm{AU}$, a maximum photodiode current of $\approx 55000 \mathrm{nA}$ is expected (i.e., all apertures of an arm are fully illuminated). This maximum value will only be reached during off-pointing when one arm is sampling the brighter inner parts of the solar disk. For comparison, illumination of only the innermost aperture (e.g. near aphelion) will generate a current of $\approx 500 \mathrm{nA}$.

\subsection{Aspect Data: Regular and Burst Mode}

The photodiode is read out continuously at a rate of $1 \mathrm{kHz}$, resulting in four signal time series corresponding to the four active areas of the quad diode. The signals are digitized with a depth of 12 bit, resulting in 4096 digital channels. A saturation limit of $62000 \mathrm{nA}$ was adopted.

As a default, 16 subsequent 12-bit values obtained at a rate of $1 \mathrm{kHz}$ are summed so that 16-bit measurements are generated at a rate of $62.5 \mathrm{~Hz}$. However, it is possible to use shorter accumulation times on command, e.g. in order to better resolve periods of rapid slewing. Instead of $16 \mathrm{~ms}$, accumulation times of 1, 2, 4, and $8 \mathrm{~ms}$ can be used, resulting in 16 bit values at higher rates (up to $1 \mathrm{kHz}$ ).

During normal operations, only a single 16-bit value is selected from each 4-second time interval. 16 of these samples are summed to a 20-bit value, for which the least significant 4 bits are then discarded. In this manner, one 16-bit value is obtained each 64 seconds. The regular aspect data thus consists of a "quintet" of data at a cadence of 64 seconds: the acquisition time and the 16-bit time-averaged output of each of the four active areas of the photodiode. This data product is transmitted to ground as part of the STIX housekeeping (HK) telemetry, and forms the primary input for the aspect solution software. Typically one set of the low-latency HK data is downlinked per day. It should be noted that the regular aspect data requires only about 1 bit per second of telemetry.

Additionally, the SAS can also provide the 16-bit measurements generated at the full cadence $(62.5 \mathrm{~Hz}$ for the default accumulation time of $16 \mathrm{~ms})$. These values are written into the same rotating buffer as the X-ray data. The flight software then periodically transfers the contents of the rotating buffer into the archive buffer for optional use as part of the burst mode aspect data. This high-resolution data set only is transmitted to ground as part of the bulk science telemetry upon user request (with time resolution selectable from $16 \mathrm{~ms}$ to $32 \mathrm{~s}$ ). This happens in case that additional data are desirable to determine or enhance an aspect solution, primarily during periods of spacecraft slewing. The determination of the need for additional data must be made on a timely basis since the on-board high-time resolution aspect data will be overwritten within a few weeks of acquisition.

\subsection{Signal Characteristics and Derivation of an Aspect Solution}

The derivation of an aspect solution is based on the analysis of the temporal evolution of the four aspect signals. When the solar image changes diameter due to the varying distance from the Sun, or when the image shifts in the image plane due to spacecraft slewing, the 
solar limb covers and uncovers different apertures. This is recorded as a step-like increase or decrease of the photodiode signal. In addition, a more gradual signal variation is observed between steps due to the solar limb darkening profile.

These signal characteristics are illustrated in Figure 5, where we have plotted simulated currents, current gradients, and relative gradients (i.e. gradient divided by signal) for the four active areas as a function of time. Here, we have assumed a Keplerian orbit with a perihelion of $0.284 \mathrm{AU}$ and an aphelion of 0.9 AU. This closely corresponds to the actual orbit of the spacecraft when the closest approach to the Sun will be reached in February 2027.

We start at $t=0$ at aphelion and approach the Sun until perihelion is reached after 83 days, and we assume that STIX is always perfectly pointed to the Sun's center. The aspect system only begins to respond after about 30 days, corresponding to a heliocentric distance of $0.82 \mathrm{AU}$. This is due to the fact that the active areas of the photodiode cannot fit more closely together and thus cannot sample the solar image which is still too small (cf. Figure 4). Note that after the closest approach in February 2027, the aphelia will actually be closer than $0.82 \mathrm{AU}$, thus allowing for continuous aspect coverage. Evenly spaced aspect coverage is available for distances smaller than $0.75 \mathrm{AU}$. The signal gradients (middle of Figure 5) clearly show the times when an aperture is being covered by the solar limb (peaks in the curves), as well as the gradual increase between aperture crossings due to the solar limb darkening profile (local minima in the curves). The relative gradients (bottom of Figure 5) show that the fractional signal increase is generally constant during most of the orbit.

The time the solar limb takes to cross an aperture is typically on the order of one day, while the interval between crossing two consecutive apertures (center to center) in one arm is typically $\approx 2$ days. The cadence of the regular aspect data is $64 \mathrm{sec}$, so there are more than 1000 samples in time for each aperture crossing. Thus, the time profile of the signal during such a crossing can be used to exactly determine the time when the solar limb is at the center of the aperture. To first order, this coincides with the maximum signal gradient, although for a precise determination the solar limb darkening profile and the varying change rate of the image diameter have to be considered, too. Achieving a desired pointing accuracy thus implies a required timing precision for the limb crossing, which is not constant over the orbit due to the varying image radius change rate. For the design goal of 4 " pointing accuracy, a maximum timing accuracy of 1 hour is sufficient. This is easily achievable given the number of samples available for each aperture crossing and the expected level of noise (see discussion in Section 3.4).

On average, one aperture crossing is measured in each of the four arms every two days, thus yielding four limb positions along two orthogonal directions within a two-day period. Since the thermal environment of the instrument is expected to be stable over this time period, this will allow the accurate determination of the position of the solar disk center with respect to the STIX imaging axis. We stress that this measurement is independent of the precise knowledge of the signal level, since it relies solely on the time profile of the signal, and not its absolute value. In this manner, the SAS is self-calibrating. In addition, comparing the measured signal strength to the predicted one provides a monitoring of the health and performance of the system.

The stability of the thermal environment over the time range of a few days suggests that this occasional calibration should be sufficient for providing the required pointing information. However, the SAS is also able to monitor the pointing continuously between the individual aperture crossings by comparing measured with simulated signals. This is possible due to the solar limb darkening profile which leads to a continuous signal variation between aperture steps (cf. Figure 5). 
Figure 5 Simulated response of the SAS as a function of time during a half-orbit, starting at $t=0$ at an aphelion of $0.9 \mathrm{AU}$ and ending at a perihelion of 0.284 AU. Shown are the diode currents (top), current gradients (middle), and relative gradients (i.e. gradient divided by signal; bottom)

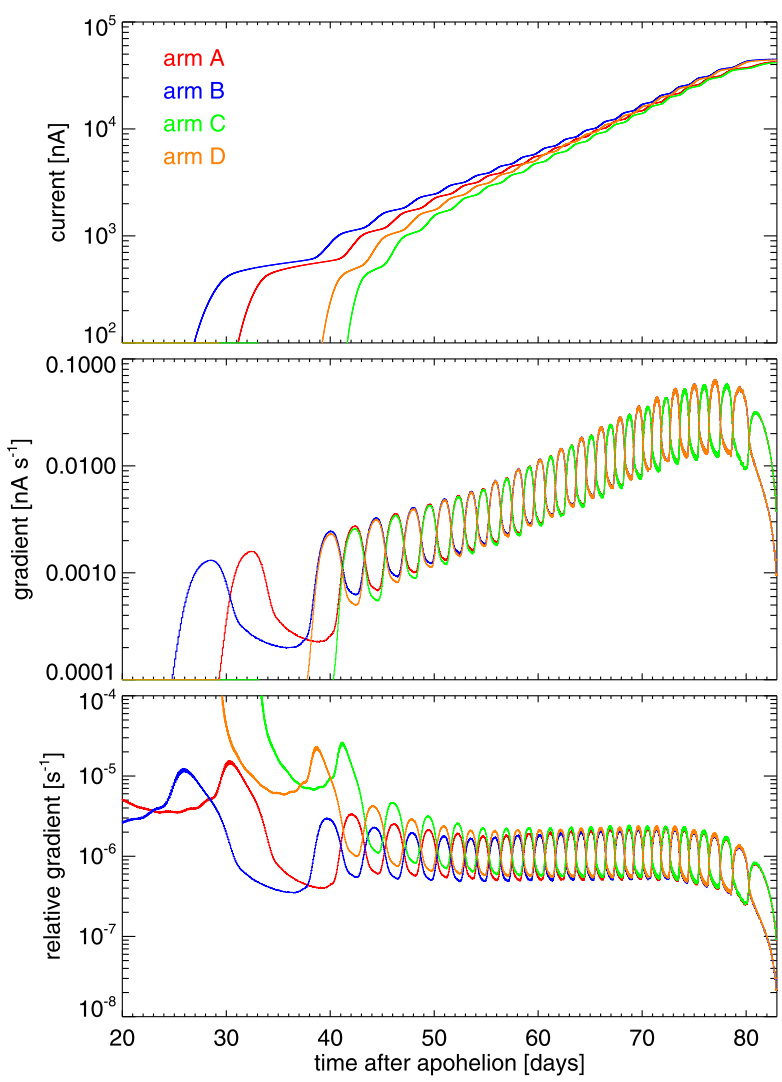

Once downlinked, the SAS signals are processed by ground analysis software which finally provides a best-fit aspect solution based on an iterative analysis technique that compares the measured to modeled signals. The derived aspect solution is then incorporated in the different STIX data products and added to a STIX aspect database. In addition to different science use cases, the aspect data is used for monitoring the status and performance of the SAS.

\subsection{Assessment of Possible in-Flight Effects}

During flight, the SAS will be subject to a performance degradation as compared to the nominal design specifications, mainly due to a decreasing $\mathrm{S} / \mathrm{N}$ ratio. This may result both from a reduction of signal strength and from an increase in noise. In the following, we provide a brief assessment of these effects.

\subsubsection{Contamination of Optical Surfaces}

Reduced light transmission through the lens and the diode glass cap will primarily result from molecular contamination, leading to a reduction of image brightness. In addition, scattering of light on the lens will result from particle contamination, with the consequence of a loss of contrast of the solar image. 


\subsubsection{Radiation-Induced Lens Degradation}

As far as lens degradation by UV radiation is concerned, the intensity at the location of the lens center is the most relevant parameter, since illumination will be at a maximum here. During most of the mission, the lens center will not see the full Sun due to the $5 \mathrm{~mm}$ aperture in the heat shield. This significantly reduces the total (bolometric) irradiance at the lens center, with a mission-averaged value of $2173 \mathrm{~W} \mathrm{~m}^{-2}$, and an intensity of $2831 \mathrm{~W} \mathrm{~m}^{-2}$ at perihelion. These values are only about twice as large as for a lens without a narrow aperture located at $1 \mathrm{AU}$. Therefore a significantly increased risk of lens degradation as compared to near-Earth missions is not expected.

\subsubsection{Radiation Damage to Photodiode}

Radiation tests simulating the mission lifetime doses (both for gamma-rays and for protons) were conducted on the photodiodes. These tests showed $\mathrm{a} \approx 14 \%$ decrease of the diode responsivity, mainly resulting from a slight darkening of the diode glass window. An increase of the dark current of $\approx 15 \mathrm{nA}$ was measured. Thus we do not expect to see a significant performance degradation due to radiation.

\subsubsection{Thermal Effects}

No significant thermal gradients are expected in the aspect lens. Changes in lens size due to temperature changes are accounted for by the lens mount (see Section 2.2.2). The only thermal effect expected will be the noise level of the photodiode and the associated aspect electronics.

\subsubsection{Misalignments}

The design of the lens mount ensures that the lens cannot tilt with respect to the optical axis, and that it cannot be displaced in the grid plane (see Section 2.2.2). Thus no change in alignment is expected. Should a constant misalignment arise from other sources, then a systematic offset will be detected between the hard X-ray flare footpoints imaged by STIX and the flare kernels or ribbons observed with the other imaging instruments on Solar Orbiter. The output of the aspect system can then be corrected for this offset because the STIX methodology ensures that any systematic error in the STIX absolute positions will tend to be the same throughout the orbit and not be temperature dependent.

\subsubsection{Influence of Sunspots}

The aspect solution is derived from a comparison of measured and modeled signals. The model assumes an idealized image brightness distribution, i.e. a radially symmetric limb darkening profile. However, photospheric structures could lead to a deviation from this simple configuration, resulting in an erroneous aspect solution. This applies particularly to sunspots, the photospheric features with the largest intensity contrast. Simulations have shown that sunspots generally have a negligible effect on the aspect solution, with one exception. Very large sunspots or spot groups near the solar limb can result in aspect errors approaching the design requirement of $\pm 4^{\prime \prime}$. In these cases, the spot will have to be modeled by the aspect analysis software, with observational constraints provided by PHI, SDO/HMI, or ground-based telescopes. 


\subsubsection{System Performance Margins}

Given that most in-flight effects discussed above (contamination, lens degradation, radiation damage, temperature effects) will lower the $\mathrm{S} / \mathrm{N}$ ratio, a large system performance margin can help to alleviate these issues. We thus have performed simulations of the SAS response adopting various $\mathrm{S} / \mathrm{N}$ factors. These simulations have shown that the regular aspect signal performance fulfills the design requirements even for a drastic reduction of the $\mathrm{S} / \mathrm{N}$ ratio. For example, the timing of the solar limb crossing one of the smallest apertures can be measured with a precision that is sufficient for fulfilling the $4^{\prime \prime}$ pointing requirement even for a 100fold decrease of the nominal $\mathrm{S} / \mathrm{N}$ ratio. While these simulations do not include further effects that may degrade SAS performance, they do show that the $\mathrm{S} / \mathrm{N}$ statistics should be a minor source of aspect error.

\section{Summary}

The STIX Aspect System (SAS) provides precise pointing information for the STIX instrument that enables ground analysis to accurately place the reconstructed X-ray images on the Sun at the 4 " level. We have designed a simple and robust system with a large performance margin that fulfills the tight constraints on all types of resources (mass, power, telemetry, etc.). This was only possible by adopting an innovative operating principle.

As designed, the SAS is in effect a small optical telescope which images the Sun and samples the image with effectively four pixels. The basic aspect data thus consist of four lightcurves which are modulated by changes of the solar image diameter, off-pointing maneuvers, and the fact that the solar image is sampled through small apertures, which introduce a step-like behavior to the lightcurves. These measurements are telemetered to ground as part of the basic housekeeping data of STIX. On ground, an aspect solution is then derived from the lightcurves. We emphasize that this is a self-calibrating method, since it does not depend on the absolute values of the photodiode output, but exclusively on its temporal evolution.

The pointing information provided by the SAS allows a meaningful comparison to be made of the reconstructed X-ray images with other space-borne or ground-based observations, and it will help to establish the magnetic connectivity of the flare with interplanetary space in general and Solar Orbiter in particular. This is a crucial prerequisite for collaborative studies with PHI, EUI, and SPICE, which represent the three other remote sensing instruments on Solar Orbiter that are capable of observing flare signatures, as well as with EPD, which will detect flare-accelerated electrons in-situ.

Acknowledgements Open Access funding provided by Projekt DEAL. We gratefully acknowledge the dedication and hard work of the scientific, technical, and administrative staff of the participating institutes and industrial partners who contributed to the STIX Aspect System. The German contribution to STIX is funded by the Bundesministerium für Wirtschaft und Technologie through Deutsches Zentrum für Luft- und Raumfahrt e. V. (DLR), grants 50 OT 0903, 1004, 1204. The work of A. W. was supported by DLR grant No. 50 QL 1701. The Swiss effort was funded by the Swiss Space Office, while the Polish contribution was supported by the Polish National Science Centre (grants 2011/01/M/ST9/06096 and 2015/19/B/ST9/02826).

Disclosure of Potential Conflicts of Interest The authors declare that they have no conflicts of interest.

Publisher's Note Springer Nature remains neutral with regard to jurisdictional claims in published maps and institutional affiliations. 
Open Access This article is licensed under a Creative Commons Attribution 4.0 International License, which permits use, sharing, adaptation, distribution and reproduction in any medium or format, as long as you give appropriate credit to the original author(s) and the source, provide a link to the Creative Commons licence, and indicate if changes were made. The images or other third party material in this article are included in the article's Creative Commons licence, unless indicated otherwise in a credit line to the material. If material is not included in the article's Creative Commons licence and your intended use is not permitted by statutory regulation or exceeds the permitted use, you will need to obtain permission directly from the copyright holder. To view a copy of this licence, visit http://creativecommons.org/licenses/by/4.0/.

\section{References}

Anderson, M., Appourchaux, T., Auchère, F., Aznar Cuadrado, R., Baraby, J., Baudin, F., Beardsley, S., Bocchialini, K., Borgo, B., Bruzzi, D., Buchlin, et al. (SPICE Consortium): 2020, The Solar Orbiter SPICE instrument: an extreme UV imaging spectrometer. Astron. Astrophys. DOI, in press.

Kosugi, T., Makishima, K., Murakami, T., Sakao, T., Dotani, T., Inda, M., Kai, K. Masuda, S., Nakajima, H., Ogawara, Y., Sawa, M., Shibasaki, K.: 1991, The Hard X-ray Telescope (HXT) for the Solar-A mission. Solar Phys. 136, 17. DOI. ADS.

Krucker, S., Hurford, G.J., Grimm, O., Kögl, S., Gröbelbauer, H.-P., Etesi, L., Casadei, D., Csillaghy, A., Benz, A.O., Arnold, N.G., Molendini, F., Orleanki, P., Schori, D., Xiao, H., Kuhar, M., Hochmuth, S., Felix, N., Schramka, F., Marcin, S., Kobler, S., Iseli, L., Dreier, M., Wiehl, H.J., Kleint, L., Battaglia, M., Lastufka, H., Sathiapal, E., Lapadula, K., Bednarzik, M., Birrer, G., Stutz, S., Wild, C., Skup, K.R., Cichocki, A., Ber, K., Rutkowski, K., Bujwan, W., Juchnikowski, G., Winkler, M., Darmetko, M., Michalska, M., Seweryn, K., Białek, A., Osica, P., Sylwester, J., Kowalinski, M., Ścisłowski, D., Siarkowski, M., Steślicki, M., Mrozek, T., Podgórski, P., Meuris, A., Limousin, O., Gevin, O., Le Mer, I., Brun, S., Strugarek, A., Vilmer, N., Musset, S., Maksimovic, M., Fárník, F., Kozacek, Z., Kašparová, J., Mann, G., Önel, H., Warmuth, A., Rendtel, J., Bauer, S.-M., Dionies, F., Paschke, J., Plüschke, D., Woche, M., Schuller, F., Veronig, A.M., Dickson, E.C.M., Gallagher, P.T., Maloney, S.A., Bloomfield, D.S., Piana, M., Massone, A.M., Benvenuto, F., Massa, P., Schwartz, R.A., Dennis, B., Van Beek, H.F., Lin, R.P.: 2020, The Spectrometer/Telescope for Imaging X-rays (STIX). Astron. Astrophys. DOI, in press.

Lin, R.P., Dennis, B.R., Hurford, G.J., Smith, D.M., Zehnder, A., Harvey, P.R., Curtis, D.W., Pankow, D., Turin, P., Bester, M., Csillaghy, A., Lewis, M., Madden, N., van Beek, H.F., Appleby, M., Raudorf, T., McTiernan, J., Ramaty, R., Schmahl, E., Schwartz, R., Krucker, S., Abiad, R., Quinn, T., Berg, P., Hashii, M., Sterling, R., Jackson, R., Pratt, R., Campbell, R.D., Malone, D., Landis, D., BarringtonLeigh, C.P., Slassi-Sennou, S., Cork, C., Clark, D., Amato, D., Orwig, L., Boyle, R., Banks, I.S., Shirey, K., Tolbert, A.K., Zarro, D., Snow, F., Thomsen, K., Henneck, R., McHedlishvili, A., Ming, P., Fivian, M., Jordan, J., Wanner, R., Crubb, J., Preble, J., Matranga, M., Benz, A., Hudson, H., Canfield, R.C., Holman, G.D., Crannell, C., Kosugi, T., Emslie, A.G., Vilmer, N., Brown, J.C., Johns-Krull, C., Aschwanden, M., Metcalf, T., Conway, A.: 2002, The Reuven Ramaty High-Energy Solar Spectroscopic Imager (RHESSI). Solar Phys. 210, 3. DOI.

Müller, D., Marsden, R.G., St. Cyr, O.C., Gilbert, H.R.: 2013, Solar orbiter. Exploring the Sun-heliosphere connection. Solar Phys. 285, 25. DOI. ADS.

Rochus, P., Auchère, F., Berghmans, D., Harra, L., Schmutz, W., Schühle, U., Addison, P., Appourchaux, T., Aznar Cuadrado, R., Baker, D., et al.: 2020, The Solar Orbiter EUI instrument: the Extreme Ultraviolet Imager. Astron. Astrophys. DOI, in press.

Rodriguez-Pacheco, J., Wimmer-Schweingruber, R.F., Mason, G.M., Ho, G.C., Sanchez-Prieto, S., Prieto, M., Martin, C., Seifert, H., Andrews, G.B., Kulkarni, S.R., Panitzsch, L., et al.: 2020, The Energetic Particle Detector. Astron. Astrophys. DOI, in press.

Skup, K.R., Cichocki, A., Graczyk, R., Michalska, M., Mosdorf, M., Nowosielski, W., Orleański, P., Przepiórka, A., Seweryn, K., Stolarski, M., Winkler, M., Sylwester, J., Kowalinski, M., Mrozek, T., Podgorski, P., Benz, A.O., Krucker, S., Hurford, G.J., Arnold, N.G., Önel, H., Meuris, A., Limousin, O., Grimm, O.: 2012, Instrument data processing unit for spectrometer/telescope for imaging X-rays (STIX). In: Photonics Applications in Astronomy, Communications, Industry, and High-Energy Physics Experiments 2012, Proc. SPIE 8454, 84540K. DOI. ADS.

Solanki, S.K., del Toro Iniesta, J.C., Woch, J., Gandorfer, A., Hirzberger, J., Alvarez-Herrero, A., Martínez Pillet, V., Perez-Grande, I., Sanchis Kilders, E., Schmidt, W., et al.: 2020, The Polarimetric and Helioseismic Imager on Solar Orbiter. Astron. Astrophys. DOI, in press. 
Zehnder, A., Bialkowski, J., Burri, F., Fivian, M., Henneck, R., Mchedlishvili, A., Ming, P., Welte, J., Thomsen, K., Clark, D., Dennis, B.R., Hurford, G.J., Curtis, D.W., Harvey, P.R., Pankow, D.H.: 2003, RHESSI imager and aspect systems. In: Keil, S.L., Avakyan, S.V. (eds.) Innovative Telescopes and Instrumentation for Solar Astrophysics, Proc. SPIE 4853, 41. DOI. ADS. 